PROCEEDINGS OF THE

AMERICAN MATHEMATICAL SOCIETY

Volume 134, Number 3, Pages 703-705

S 0002-9939(05)07925-6

Article electronically published on September 28, 2005

\title{
ADMISSIBLE MEASURES IN ONE DIMENSION
}

\author{
JANA BJÖRN, STEPHEN BUCKLEY, AND STEPHEN KEITH
}

(Communicated by Andreas Seeger)

\begin{abstract}
In this note we show that $p$-admissible measures in one dimension (i.e. doubling measures admitting a $p$-Poincaré inequality) are precisely the Muckenhoupt $A_{p}$-weights.
\end{abstract}

In the last two decades it has been observed that much of the theory for $p$ harmonic functions can be extended to the situation when the Lebesgue measure on $\mathbf{R}^{n}$ is replaced by another measure satisfying certain conditions; see e.g. FabesKenig-Serapioni [2] and Heinonen-Kilpeläinen-Martio [4. More precisely, Theorem 2 in Hajłasz-Koskela [3] and Theorem 5.2 in Heinonen-Koskela [5] show that the following two conditions are exactly what is needed for the theory to go through.

Definition 1. A measure $\mu$ on $\mathbf{R}^{n}$ is called $p$-admissible with $p \geq 1$ if it satisfies the following two conditions:

- It is doubling, i.e. there is a constant $C>0$ such that

$$
\mu(2 B)<C \mu(B)
$$

for all balls $B \subset \mathbf{R}^{n}$, where $2 B$ denotes the ball concentric with $B$ and with twice the radius.

- It admits the weak p-Poincaré inequality, i.e. there exist $C>0$ and $\lambda \geq 1$ such that

$$
\frac{1}{\mu(B)} \int_{B}\left|u-u_{B}\right| d \mu \leq C r\left(\frac{1}{\mu(\lambda B)} \int_{\lambda B}|\nabla u|^{p} d \mu\right)^{1 / p}
$$

holds whenever $B$ is a ball with radius $r$ and $u$ is, say, a locally Lipschitz function on $\lambda B$. Here and in what follows, $u_{B}=\mu(B)^{-1} \int_{B} u d \mu$.

Received by the editors July 6, 2004

2000 Mathematics Subject Classification. Primary 26D10; Secondary 46 E35.

Key words and phrases. Admissible measure, Muckenhoupt $A_{p}$-weight, doubling measure, Poincaré inequality.

The first author was supported by the Swedish Research Council and Gustaf Sigurd Magnuson's fund of the Royal Swedish Academy of Sciences, and completed this research while at Lund University.

The second author was partially supported by Enterprise Ireland.

The third author was supported by the Australian Research Council.

(C)2005 American Mathematical Society Reverts to public domain 28 years from publication 
The Hölder inequality implies that every $p$-admissible measure is also $p^{\prime}$-admissible for all $p^{\prime}>p$. Conversely, by a recent result due to Keith-Zhong [6], every $p$-admissible measure with $p>1$ is also $p^{\prime}$-admissible for some $p^{\prime}<p$.

Unfortunately, in many situations, the Poincaré inequality is rather difficult to verify. In this note we give a more straightforward characterization of admissible measures in one dimension, namely we prove the following result.

Theorem 2. Let $\mu$ be a measure on $\mathbf{R}$ and let $p \geq 1$. Then $\mu$ is $p$-admissible in $\mathbf{R}$ if and only if $d \mu=w d x$ and $w$ is a Muckenhoupt $A_{p}$-weight.

Definition 3. A nonnegative function $w$ on $\mathbf{R}^{n}$ is a Muckenhoupt $A_{p}$-weight with $p \geq 1$, if for some $C>0$ and all balls $B \subset \mathbf{R}^{n}$,

$$
\frac{1}{|B|} \int_{B} w d x< \begin{cases}C\left(\frac{1}{|B|} \int_{B} w^{1 /(1-p)} d x\right)^{1-p} & \text { for } p>1 \\ C \underset{B}{\operatorname{essinf} w} & \text { for } p=1\end{cases}
$$

where $|B|$ denotes the Lebesgue measure of $B$.

Remark 4. Note that Theorem 2 fails in $\mathbf{R}^{n}$ if $n \geq 2$. By e.g. Corollary 15.35 in Heinonen-Kilpeläinen-Martio 4, the measures $d \mu=|x|^{\alpha} d x$ with $\alpha>0$ are $p$-admissible in $\mathbf{R}^{n}, n \geq 2$, for all $p>1$, but belong to $A_{p}$ if and only if $p>1+n \alpha$.

To prove Theorem 2, we use the following lemma. For a proof, see the corollary on p. 200 in Stein [7].

Lemma 5. Let $\mu$ be a nonnegative Borel measure on $\mathbf{R}^{n}$ and assume that there exists $C>0$ such that

$$
\frac{1}{|B|} \int_{B} f(x) d x \leq C\left(\frac{1}{\mu(B)} \int_{B} f^{p} d \mu\right)^{1 / p}
$$

for all balls $B \subset \mathbf{R}^{n}$ and all nonnegative measurable functions $f$ on $B$. Then $\mu$ is absolutely continuous with respect to the Lebesgue measure, $d \mu=w d x$ and $w$ is a Muckenhoupt $A_{p}$-weight.

In the rest of this note, $C>0$ denotes a constant whose value may vary with each usage but depends only on the doubling constant of $\mu$ and on the constants in the Poincaré inequality.

Proof of Theorem 2, The "if" part of the theorem is proved e.g. in Theorem 15.21 in Heinonen-Kilpeläinen-Martio [4]. To prove the "only if" part, let $f \geq 0$ be a measurable function supported on an interval $I \subset \mathbf{R}$. For $k \in \mathbf{N}$, let $f_{k}=\min \{f, k\}$ and

$$
u_{k}(x)=\int_{-\infty}^{x} f_{k}(t) \chi_{I}(t) d t
$$

Then $u_{k}$ is Lipschitz and we can test the weak $p$-Poincaré inequality with it on the concentric double $2 I$ of $I$. On the right-hand side we have

$$
C|I|\left(\frac{1}{\mu(2 \lambda I)} \int_{2 \lambda I}\left(u_{k}^{\prime}\right)^{p} d \mu\right)^{1 / p} \leq C|I|\left(\frac{1}{\mu(I)} \int_{I} f^{p} d \mu\right)^{1 / p} .
$$

To estimate the left-hand side in the Poincaré inequality, let $I_{-}$and $I_{+}$denote the parts of $2 I \backslash I$ lying to the left and to the right of $I$, respectively. Then $u_{k}=0$ 
on $I_{-}$and

$$
u_{k}=\int_{I} f_{k}(x) d x
$$

on $I_{+}$. Using the doubling property of $\mu$, the left-hand side in the Poincaré inequality can be estimated as

$$
\begin{aligned}
\frac{1}{\mu(2 I)} \int_{2 I}\left|u_{k}-\left(u_{k}\right)_{2 I}\right| d \mu & \geq \frac{1}{\mu(2 I)}\left(\int_{I_{-}}\left(u_{k}\right)_{2 I} d \mu+\int_{I_{+}}\left(\int_{I} f_{k}(x) d x-\left(u_{k}\right)_{2 I}\right) d \mu\right) \\
& \geq C \int_{I} f_{k}(x) d x .
\end{aligned}
$$

Inserting both estimates into the weak $p$-Poincaré inequality, together with the monotone convergence theorem, shows that the assumptions in Lemma [5] are satisfied and hence $d \mu=w d x$ with $w$ a Muckenhoupt $A_{p}$-weight.

Remark 6. If we knew a priori that $\mu$ is absolutely continuous with respect to the Lebesgue measure, then Theorem 2 could also be obtained after some calculation from Theorem 1.4 in Chua-Wheeden [1].

\section{REFERENCES}

[1] Chua, S. K. and Wheeden, R. L., Sharp conditions for weighted 1-dimensional Poincaré inequalities, Indiana Univ. Math. J. 49 (2000), 143-175 MR1777034 (2001h:26021)

[2] Fabes, E. B., Kenig, C. E. and Serapioni, R. P., The local regularity of solutions of degenerate elliptic equations, Comm. Partial Differential Equations 7 (1982), 77-116 MR0643158 (84i:35070)

[3] Hajłasz, P. and Koskela, P., Sobolev meets Poincaré, C. R. Acad. Sci. Paris Sér. I Math. 320 (1995), 1211-1215 MR1336257(96f:46062)

[4] Heinonen, J., Kilpeläinen, T. and Martio, O., Nonlinear Potential Theory of Degenerate Elliptic Equations, Oxford Univ. Press, Oxford, 1993 MR.1207810 (94e:31003)

[5] Heinonen, J. and Koskela, P., Weighted Sobolev and Poincaré inequalities and quasiregular mappings of polynomial type, Math. Scand. 77 (1995), 251-271 MR.1379269 (97e:30039)

[6] Keith, S. and Zhong, X., The Poincaré inequality is an open ended condition, Preprint, Jyväskylä 2003

[7] Stein, E. M., Harmonic Analysis: Real-variable Methods, Orthogonality, and Oscillatory Integrals, Princeton Mathematical Series 46, Princeton Univ. Press, Princeton, N. J., 1993 MR.1232192 (95c:42002)

Department of Mathematics, Linköping University, SE-581 83 Linköping, Sweden

E-mail address: jabjo@mai.liu.se

Department of Mathematics, National University of Ireland, Maynooth, County KILDARE, IRELAND

E-mail address: sbuckley@maths.may.ie

Centre for Mathematics and its Application, Australian National University, CanBerra, ACT 0200, Australia

E-mail address: keith@maths.anu.edu.au 